\title{
Clinical applications of diffusion-weighted sequence in brain imaging: beyond stroke
}

\author{
Siddhartha Gaddamanugu ${ }^{1} \cdot$ Omid Shafaat $^{2} \cdot$ Houman Sotoudeh $^{3} \cdot$ Amir Hossein Sarrami $^{4} \cdot$ Ali Rezaei $^{3}$. \\ Zahra Saadatpour ${ }^{3} \cdot$ Aparna Singhal $^{3}$
}

Received: 7 July 2021 / Accepted: 10 September 2021 / Published online: 1 October 2021

This is a U.S. government work and not under copyright protection in the U.S.; foreign copyright protection may apply 2021

\begin{abstract}
Diffusion-weighted imaging (DWI) is a well-established MRI sequence for diagnosing early stroke and provides therapeutic implications. However, DWI yields pertinent information in various other brain pathologies and helps establish a specific diagnosis and management of other central nervous system disorders. Some of these conditions can present with acute changes in neurological status and mimic stroke. This review will focus briefly on diffusion imaging techniques, followed by a more comprehensive description of the utility of DWI in common neurological entities beyond stroke.
\end{abstract}

Keywords Diffusion-weighted imaging · Infection · Trauma · Demyelinations · Brain tumors, Epilepsy

\begin{tabular}{llll}
\multicolumn{2}{l}{ Abbreviations } & COVID-19 & Coronavirus 2019 \\
DWI & Diffusion-weighted imaging & TBI & Traumatic brain injuries \\
RSNA & Radiological Society of North America & MS & Multiple sclerosis \\
ADC & Apparent diffusion coefficient & PRES & Posterior reversible encephalopathy \\
CSF & Cerebrospinal fluid & ODM & Osmotic demyelination \\
CNS & Central nervous system & CLOCC & Cytotoxic lesions of the corpus callosum \\
FA & Fractional anisotropy & PCA & Posterior cerebral artery \\
GBM & Glioblastoma multiforme & SE & Status epilepticus \\
MR & Magnetic resonance & rCBV & Relative cerebral blood volume \\
HIV & Human immunodeficiency virus & PCNSL & Primary CNS lymphoma \\
CJD & Creutzfeldt Jakob disease & & \\
EEG & Electroencephalography & & \\
SCJD & Sporadic Creutzfeldt Jakob disease & & \\
vCJD & Variant Creutzfeldt Jakob disease & & \\
FLAIR & Fluid-attenuated inversion recovery & & \\
HSV & Herpes simplex virus & &
\end{tabular}

Siddhartha Gaddamanugu

sgaddamanugu@uabmc.edu

Omid Shafaat

omid.shafaat@yahoo.com

Houman Sotoudeh

hsotoudeh@uabmc.edu

Amir Hossein Sarrami

sarramiradio@gmail.com

Ali Rezaei

arezaei@uabmc.edu

Zahra Saadatpour

zsadaatpour@uabmc.edu
Aparna Singhal

asinghal@uabmc.edu

1 Department of Radiology, Birmingham VA Medical Center, University of Alabama at Birmingham, 700 S. 19th Street, Birmingham, AL 35233, USA

2 The Russell H. Morgan Department of Radiology and Radiological Science, Johns Hopkins University School of Medicine, Baltimore, MD, USA

3 Department of Radiology, University of Alabama at Birmingham, Birmingham, AL, USA

4 Department of Radiology, University of Semnan, Semnan, Iran 


\section{Introduction}

\section{History and technical aspects of diffusion imaging}

Diffusion-weighted imaging (DWI) was first presented by Denis LeBihan, MD, Ph.D., at the Radiological Society of North America (RSNA) annual meeting in 1985. Although initially utilized in liver imaging, it was soon incorporated in neuroimaging. It became the first imaging technique using physiology to reveal imaging characteristics and found utility in the diagnosis of stroke imaging and abscess $[1,2]$.

DWI characterizes the water motion in the human body. Normal water motion in the brain is predominantly random or Brownian, with no direction predilection. This is termed isotropic diffusion. However, myelin sheaths' orientation can create a preferential movement of water molecules parallel to the fibers and hence an "anisotropic diffusion." Diffusion imaging involves a slight modification of T2-weighted sequences. Two diffusion sensitizing gradients of equal strength (termed the b value) are applied in opposing directions before and after a $180^{\circ}$ refocusing pulse (Fig. 1). The first gradient pulse causes a phase shift, and the second pulse will "rephase" the initial phase shift. For stationary water molecules, there is no loss of phase and signal by the gradient pulses. Moving water molecules have a net dephasing effect by the gradient pulses and will result in phase shift and signal loss [3]. DWI needs to be acquired rapidly as adjacent bulk motion from vessels can degrade the study. Echo planar imaging (EPI) facilitated DWI (EPI-DWI) provides rapid generation of diffusion gradients and can generate 1 slice in $100 \mathrm{~ms}$; however, EPI-DWI suffers from image degradation due to each echo being acquired at a different echo time, resulting in image blurring. A Turbospin (TS-DWI) technique introduces a new RF refocusing pulse and has longer T2 relaxations than the EPI-DWI technique. This results in less magnetic field susceptibility and image blurring [4]. The disadvantages of TS-DWI techniques include a longer scan time [5]. EPI-DWI techniques are limited due to bowel peristalsis. EPI-DWI technique remains the standard of choice for brain imaging.

DWI constitutes the influence of T2 signal on the qualitative assessment of diffusion. Apparent diffusion coefficient (ADC) images provide the opportunity to estimate the magnitude of water diffusion (in $\mathrm{mm}^{2} / \mathrm{sec}$ ) by calculating the net diffusion of water both before and after application of diffusion gradients based on the following formula $\mathrm{ADC}=-\mathrm{b}$ $\ln (\mathrm{DWI} / \mathrm{b} 0)$. In this equation, $b$ represents the applied gradient strengths, and $b 0$ is the signal with no diffusion gradients applied. Since the ADC calculates the net diffusion, it is not influenced by T2 effects. Since a negative sign precedes the calculation, decreased diffusion is demonstrated as a dark signal [6].

Higher $b$ values result in greater contrast; however, they also result in lost absolute signal creating a low

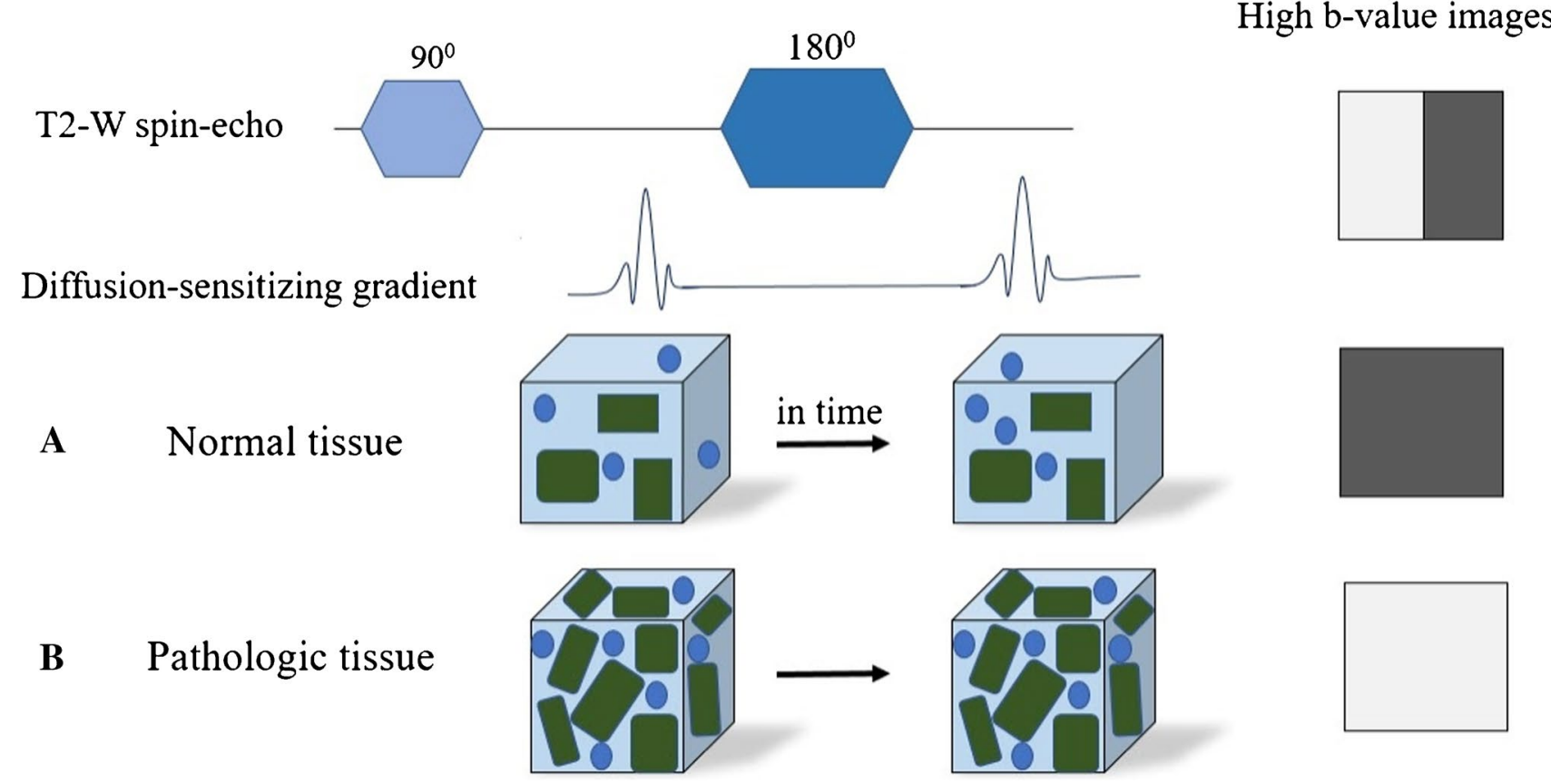

Fig. 1 Application of 2 gradients in equal but opposite directions in a T2 sequence results in net signal loss in moving water molecules but bright signal from water with restriction of motion 
signal-to-noise ratio [7]. Diffusion bright signal is considered "true" when there is a corresponding dark ADC signal (suggesting less T2 effects). "False" diffusion signal or T2 shine through denotes bright DWI signal with accompanying bright ADC signal.

Within the brain parenchyma, there are variable diffusion signals with white matter displaying increased diffusion compared to the cortex. Normal average ADC values of white matter and cortex are $0.84 \times 10^{-3} \mathrm{~mm} / \mathrm{sec}$ and $0.76 \times 10^{-3} \mathrm{~mm} / \mathrm{sec}$, respectively. ADC values of cerebrospinal fluid (CSF) is about $2.40 \pm 4.40 \times 10^{-3} \mathrm{~mm} / \mathrm{sec}$ [8].

Axial imaging is the preferred plane for brain imaging. Thinner slices $(3 \mathrm{~mm})$ and sagittal plane imaging increase the sensitivity for detection of small brain stem strokes [9]. Combined coronal and sagittal DWI also improve the sensitivity for detection of brain stem strokes [10]. However, thinner slices can increase scan time. In an effort to reduce scan time, the parallel technique has been implemented with thinner slices $(3 \mathrm{~mm})$, where complex spatial encoding by the receiver coils is partially replaced, thus reducing the scan time [11].

\section{Bacterial brain abscess}

Brain abscesses can be bacterial, parasitic, or fungal. A bacterial infection constitutes four stages: (1) early cerebritis (days 1-4); (2) late cerebritis (days 4-10); (3) early capsule formation (days 11-14); and (4) late capsule formation (after 14 days). The early cerebritis can show patchy areas of diffusion restriction which progress with late cerebritis. On conventional imaging, a mature abscess would demonstrate a rim enhancing lesion with a T2 hypointense rim and associated vasogenic edema, appearing similar to cystic or necrotic neoplasms and subacute hematomas. However, a pyogenic abscess would show restricted diffusion with decreased signal on the ADC map due to the presence of inflammatory cells and bacteria, which restricts the water molecules diffusion (Fig. 2A). An abscess cavity has lower ADC and higher fractional anisotropy (FA) in contrast to cystic glioblastomas and metastases. It has been reported that low ADC values can differentiate abscess from cystic glioblastoma multiforme (GBM) and metastasis with sensitivity and specificity of 96\% [12], possibly secondary to high viscosity from inflammatory macrophages, white blood cells, and decaying or viable macrophages [13]. [12, 14]. DWI is more sensitive than other magnetic resonance (MR) sequences to differentiate the abscesses from cystic neoplasms [15]. DWI and ADC maps also can help to differentiate the post-surgical abscesses from the spontaneous ones; the ADC value of the spontaneous abscesses is usually lower than post-surgical abscesses [16].
Diffusion imaging is the most sensitive technique for detecting extension of infection into the ventricles spontaneously or from ruptured abscesses into the ventricles. Debris within the ventricles is the most common finding and best identified as a bright signal on DWI [17, 18] (Fig. 2B). [19]. $[20,21]$.

\section{Fungal brain abscess}

Fungal abscesses have variable diffusion signals. The fungal abscess cavity can have circumferential projections representing mycelia which can show low ADC while the center demonstrates increased diffusion and higher ADC values [22] (Fig. 2C). The mean ADC value of bacterial abscesses $\left(0.11-0.76 \times 10^{-3} \mathrm{~mm}^{2} / \mathrm{sec}\right)$ is lower than fungal abscesses $\left(0.35-0.97 \times 10^{-3} \mathrm{~mm}^{2} / \mathrm{sec}\right)[23]$.

\section{Toxoplasmosis}

Toxoplasmosis can show variable diffusion signal (Fig. 2D), and DWI signal changes cannot accurately differentiate a toxoplasma abscess from lymphoma, which is another common cause of ring-enhancing lesion in immunocompromised/human immunodeficiency virus (HIV) infected patients [24]. It is believed that the " eccentric target sign" (an enhancing mural nodule within the rim-enhancing lesions) is pathognomonic for toxoplasmosis [25].

\section{Neurocysticercosis}

Neurocysticercosis is a common parasitic infection worldwide. It can present in various radiological stages. Neurocysticercosis can present as vesicular, vesicular nodular, granular nodular, and finally calcified nodular stages in order of appearance. The first two stages present as cysts of varying sizes, usually in the cortex or subarachnoid space or rarely the ventricles. In vesicular stage, the cysts contain viable numbers of parasites in the vesicular stage with increased diffusion, similar to CSF [26]. In the vesicular nodular stage, the parasites are dying, inciting an inflammatory reaction, and the "entire" cyst content becomes turbid and can demonstrate different DWI signals. DWI hyper signal has been reported in 5\% of cysts in the colloidal vesicular stage and $3 \%$ of the cysts in the granular nodular phase [27]. DWI is more helpful to detect the scolex. The scolex is a small eccentric DWI hyperintense dot/curvilinear structure seen in $29 \%$ of lesions in the vesicular phase, $19 \%$ of colloidal vesicular, $22 \%$ of subarachnoid lesions, and $14 \%$ of the intraventricular lesions [27]. 
Fig. 2 A cerebral pyogenic abscess demonstrating diffusion restriction (A). Ventriculits demonstrates as bright signal in the right lateral ventricle (B) Cerebral blastomycosis $(\mathbf{C})$ abscess in the midbrain with no diffusion restriction typical of fungal abscesses. Cerebral toxoplasmosis: cerebral abscess in the left cerebellum showing concentric layers of varying diffusion typical of toxoplasmosis (D)

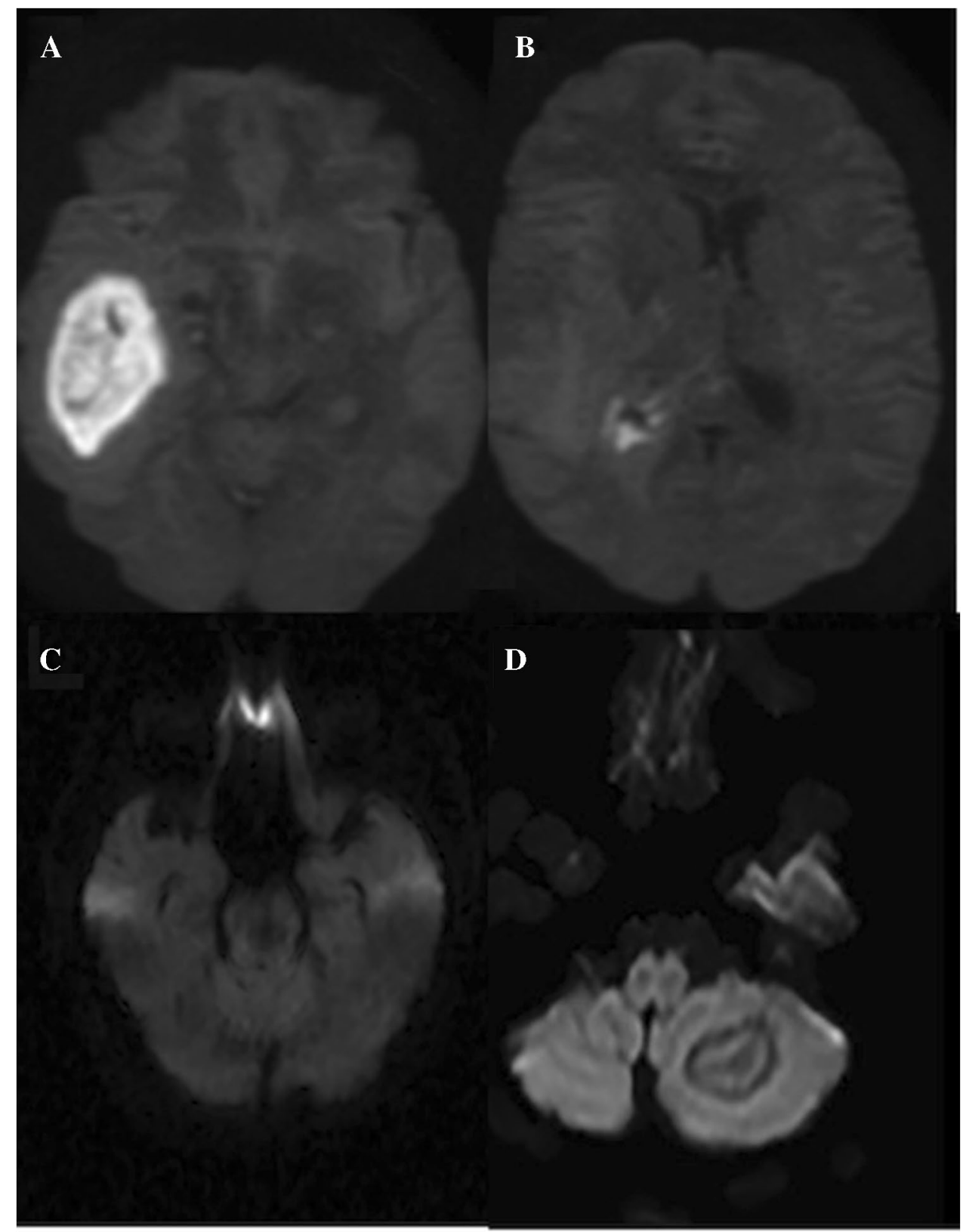

\section{Creutzfeldt Jakob disease (CJD)}

CJD is a CNS infection resulting from prion particles. Intracellular prion deposition causes cellular vacuolization, which is felt to cause diffusion changes. Diffusion changes occur early in the disease course and can precede electroencephalography, CSF, and the typical clinical symptoms [28]. CJD is known to have subtypes, with the most common type called sporadic (sCJD). Variant (vCJD) is attributed to the spread from animals (mostly cows). In SCJD, there is restricted diffusion in the basal ganglia, insular cortex, hippocampus, and cerebral cortex (Fig. 3C). While the distribution is bilateral, it also can be asymmetric. The perirolandic cortex is typically spared. Cerebellar atrophy can happen, but diffusion restriction of the cerebellum is rare. While both fluid-attenuated inversion recovery (FLAIR) and DWI are sensitive for detecting basal ganglia abnormalities, cortical signal changes are better identified on DWI (Fig. 3C and $\mathrm{D}$ ).As the disease progresses, resultant gliosis causes normalization or increased diffusion [29]. In vCJD, diffusion signal changes are classically described in the posterior thalamus (termed Pulvinar sign) or dorsomedial thalamus (termed Hockey stick sign) [29]. In SCJD, DWI hyper signal intensity is rarely seen in the precentral gyrus, and isolated DWI hyperintensity of limbic system rarely happens [28, 30]. DWI is the most diagnostic test to differentiate CJD from its differential diagnosis (Alzheimer's disease, dementia with Lewy bodies, genetic neurodegenerative disorders, 
Fig. 3 A 61-year-old woman with a history of SLE, RA, Sjogren syndrome, and hypothyroidism presents with left hemiparesis, altered mental status and right frontotemporal seizures. CSF studies yielded a diagnosis of HSV. Initial DWI (A) revealed cytotoxic edema in the insular cortex which progressed to involve the hippocampus and cingulate gyrus on a MRI performed 8 days later (B) A 45-year-old female with rapidly progressive cognitive decline over the past 6 months. DWI (C) demonstrates symmetric diffusion abnormality in the cerebral cortex with corresponding low ADC (D) suggesting CJD. The patient was confirmed to have prion disease

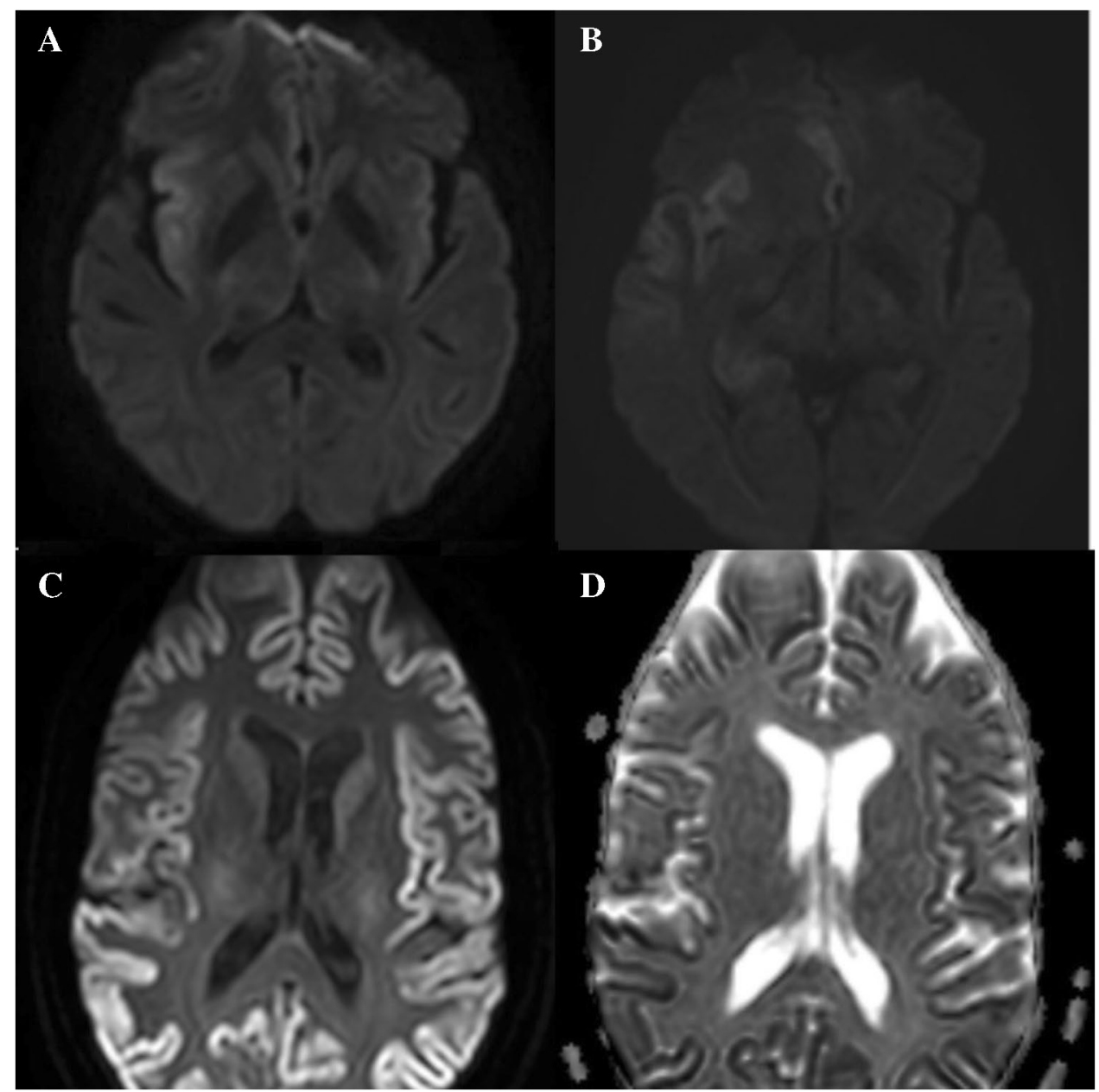

immune-mediated encephalopathy, lymphoma, hepatic encephalopathy, and progressive multifocal leukoencephalopathy)[31].

\section{Application of DWI in the diagnosis of encephalitis}

Encephalitis is defined as inflammation of the brain [32, 33]. It is always challenging to find the cause of encephalitis, and the etiology usually remains unknown. The two most common causes of encephalitis include infections and immune-mediated conditions. Infectious encephalitis is most commonly caused by viruses like herpes simplex virus (HSV) type 1, varicella-zoster virus, enterovirus [34], and recently coronavirus 2019 (COVID-19)[35]. Immunemediated encephalitis includes paraneoplastic encephalitis syndrome and encephalitis syndrome associated with antibodies against neuronal cells (i.e., autoimmune encephalitis) [12]. In the early phase of encephalitis, diagnosis is very challenging; it is based on clinical presentation, laboratory analysis, electrophysiologic assessment, and neuroimaging (i.e., fever, CSF pleocytosis, EEG changes, and MRI abnormality, respectively). Conventional MR sequences may be normal or nonspecific in the early stage of encephalitis (i.e., high FLAIR signal in cortical and subcortical regions) [36]. DWI can be useful in both infectious and autoimmune encephalitis.

In infectious encephalitis, such as viral encephalitis, DWI shows high sensitivity, which can be used in early diagnosis [37-39] (Fig. 3B). DWI can diagnose HSV-1 encephalitis as early as $40 \mathrm{~h}$ after the onset of symptoms and may be more sensitive than the T2-weighted sequence (Fig. 3A and B) [40]. In other less common causes of infectious encephalitis, such as enterovirus 71, DWI is also a reliable technique for detecting encephalitis. These studies have shown that early reversible cytotoxic edema is a feature of viral encephalitis [36]. In immune-mediated encephalitis, especially in limbic encephalitis, diffusion changes have been reported along with FLAIR abnormalities [41-43]. Pathology in limbic encephalitis is felt to be due to vasogenic edema, and the DWI hyper signal intensities are felt to be due to $\mathrm{T} 2$ shine through effect. 


\section{Trauma (axonal injury)}

DWI is a promising tool for depicting post-traumatic brain injuries (TBI) and outcome prediction in adults and pediatrics [44-46]. It can be used to detect axonal injuries not visualized on FLAIR, T2-weighted, and T2*-weighted GRE sequences $[44,47]$. The volume of lesions depicted by DWI can predict the clinical outcome; moreover, the presence of DWI lesions in the corpus callosum are the most important predictive MRI variable in patients with severe TBI [48]. Diffuse axonal injury can present with DWI bright foci (Fig. 4A) with ADC dark signal (cytotoxic edema) or ADC bright signal (vasogenic edema) and may also contain hemorrhage (Fig. 4B). These changes can present in the first $24 \mathrm{~h}$, and patients with cytotoxic edema are associated with more severe TBI [49]. Hemorrhagic lesions on SWI or GRE recalled images may not display DWI changes and are associated with moderate TBI [48].

Another distinct injury to the brain is cerebral fat embolism (CFE) related to long bone fractures or sickle cell anemia. Various patterns of CFE are identified on imaging depending on the time interval from initial neurological symptoms. In the acute stage, there are tiny diffusion abnormalities scattered in the deep cerebral white matter and basal ganglia (type 1: starfield pattern). In the subacute phase, diffusion restriction is more confluent in the periventricular white matter (type 2A: confluent cytotoxic edema). [50]

\section{Demyelination}

Demyelinating lesions, in particular multiple sclerosis (MS) plaques, show imaging diversity both on conventional sequences and on DWI $[51,52]$. Application of DWI in MS is of benefit to establish a differential diagnosis with other abnormalities, such as tumors, acute infarction, and inflammatory/infectious conditions [53, 54]. In multiple sclerosis, the ADC value is different among various lesions, and they tend to change over a short period of time. ADC values range from $1.03 \times 10^{-3} \mathrm{~mm}^{2} / \mathrm{sec}$ in the enhancing portion of the active lesion to $2.14 \times 10^{-3} \mathrm{~mm}^{2} / \mathrm{sec}$ in the cystic lesion. Decreased ADC value is the marker of the acute demyelination in MS (Fig. 5A and B), followed by rapid normalization of the ADC value secondary to vasogenic edema [55]. Variable ADC values among different demyelinating lesions can potentially differentiate MS from neoplasms, infections, and ischemic lesions [53]. The other application of DWI is the depiction of the demyelination plaques earlier than other MR sequences. DWI findings can precede contrast enhancement and biomarkers for an acute attack, disease severity, and clinical deterioration $[53,55,56]$. There can be occasionally restricted diffusion in a demyelinating plaque before $\mathrm{T} 2$ signal changes or enhancement appears. Once conventional sequences for MS are positive, the DWI hyper signal intensity is because of T2 shine through effect (Fig. 5C and D).

Chronic MS plaques demonstrate increased diffusion (Figs. 5E and F). However, diffusion changes in MS are subject to variability resulting in varying confidence among interpreters to use DWI changes for age determination of the plaque.
Fig. 4 A 70-year-old patient with diffuse axonal injury, multiple hemorrhagic foci $(\mathbf{B})$ at gray white matter demonstrating restricted diffusion $(\mathbf{A})$, which is associated with greater degree of traumatic brain injury

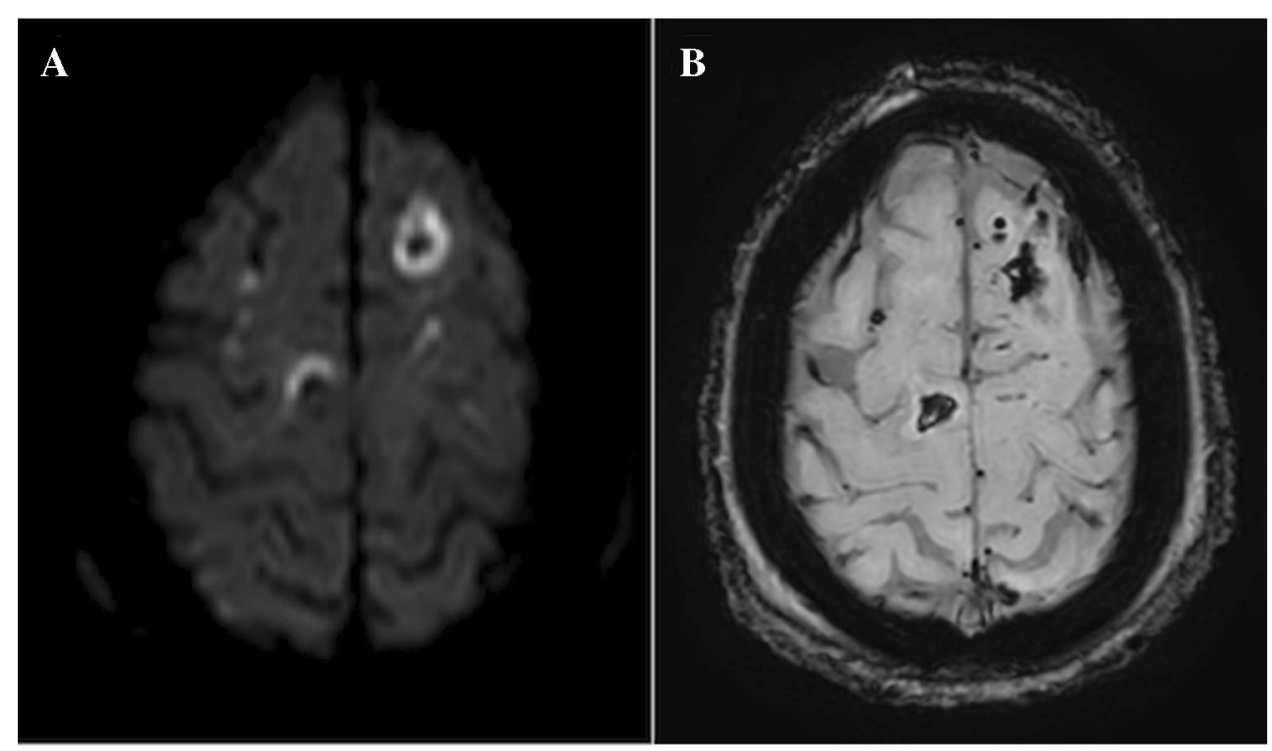


Fig. 5 A 40-year-old male with active demyelination: Diffusion restriction (A on ADC) and enhancement $(\mathbf{B})$ at the margin of the demyelinating plaque with increased diffusion elsewhere on ADC. An 18-yearold female patient with MS: T2 shine through on DWI (C) and ADC (D). A 40-year-old male with chronic demyelinating plaque in the posterior right frontal lobe demonstrating increased diffusion ( $\mathbf{E}$ and $\mathbf{F}$ )

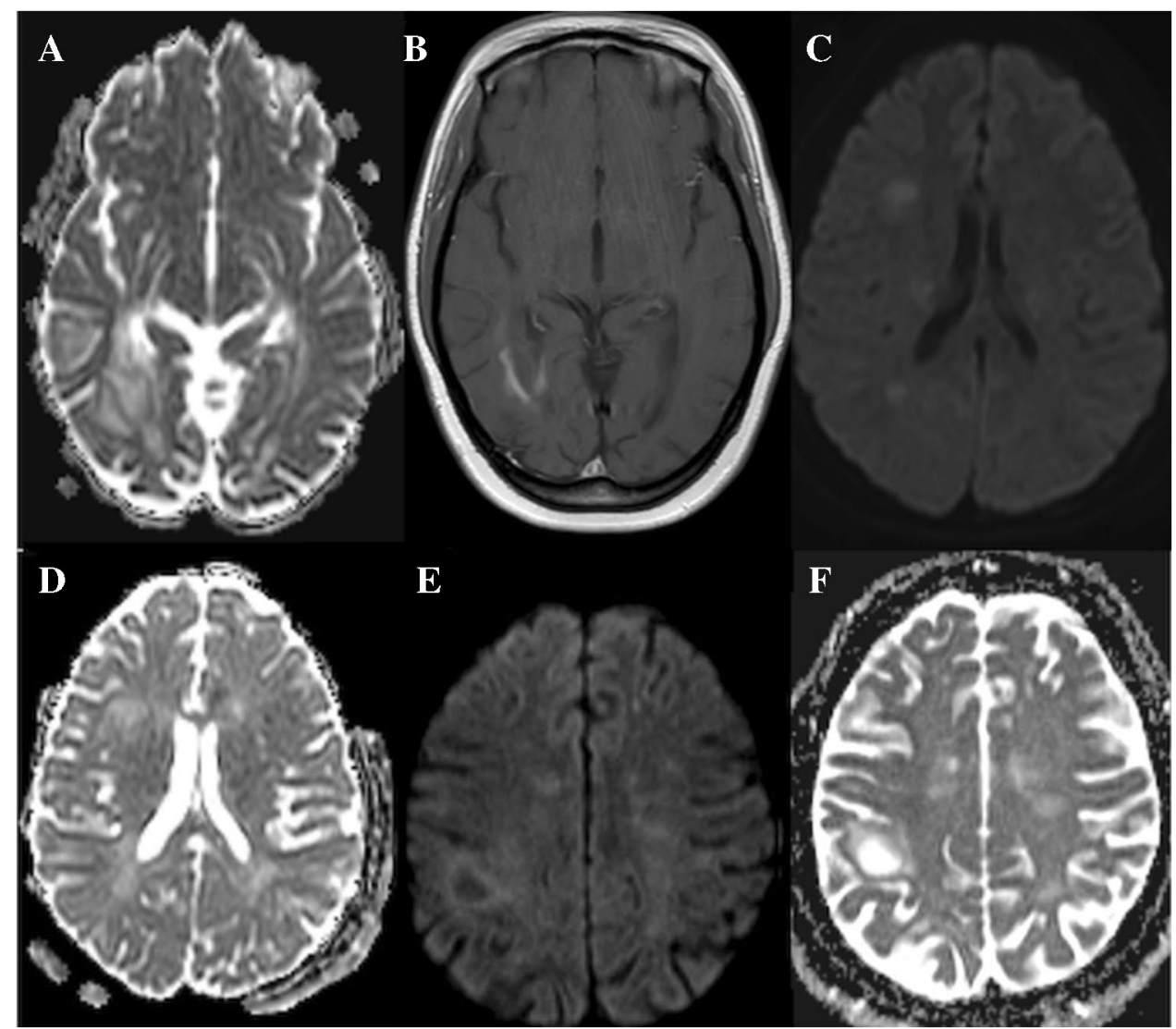

\section{Metabolic (includes hypoxic-ischemic encephalopathy, reversible cytotoxic lesion of corpus callosum)}

Diffusion imaging has shown remarkable value in diagnosing metabolic conditions.

\section{Toxic encephalopathies}

Common exogenous toxins resulting in encephalopathy are carbon monoxide (CO), opioids, methotrexate, and metronidazole. Endogenous toxic encephalopathy includes posterior reversible encephalopathy (PRES), acute hepatic encephalopathy, and uremic encephalopathy. All toxic encephalopathies typically cause diffusion changes from either cytotoxic or vasogenic edema.

Carbon monoxide poisoning causes symmetrical bright $\mathrm{T} 2$ signals in the globus pallidus and periventricular white matter. However, these changes initially appear as restricted diffusion (Fig. 6A), in the hyperacute phase, and diffusion changes rapidly normalize as T2 changes persist [57].

Opioids cause brain injury mainly to the globus pallidus, cerebral, and cerebellar white matter with early diffusion restriction. Methotrexate and metronidazole cause reversible diffusion and T2 signal changes. Methotrexate typically causes diffusion signal changes in the cerebral white matter, while Metronidazole favors the dentate nucleus and splenium of the corpus callosum (Fig. 6B). DWI abnormalities in metronidazole toxicity involving the dentate nuclei typically have a bright ADC signal suggesting T2 shine through [57].

Hepatic encephalopathy and uremic encephalopathy can cause edema in the basal ganglia, cortex, and white matter manifesting as T2/FLAIR hyperintensity. There is a greater degree of vasogenic edema in these patients, and hence, diffusion changes are not present in all cases [58]. The vasogenic edema and increased ADC value in chronic hepatic encephalopathy have been attributed to chronic astrocytic swelling, increased interstitial fluid, or chronic demyelination [59]. On the other hand, in acute hepatic encephalopathy, DWI hyper signal intensities tend to be true because of intramyelinic edema, intracellular edema, acute astrocytic swelling, or oligodendroglial injury [59]. DWI hyper signal intensities in acute hepatic encephalopathy usually involve the thalami, periventricular white matter, brainstem, and cerebral cortex. There is an association between the plasma ammonia level and extension of DWI hyper signal intensities. Also, diffusion restriction in acute hepatic encephalopathy can be reversible [59-61]. On DWI and FLAIR sequences, symmetrical involvement of the insula (most common), thalamus, posterior limbs 


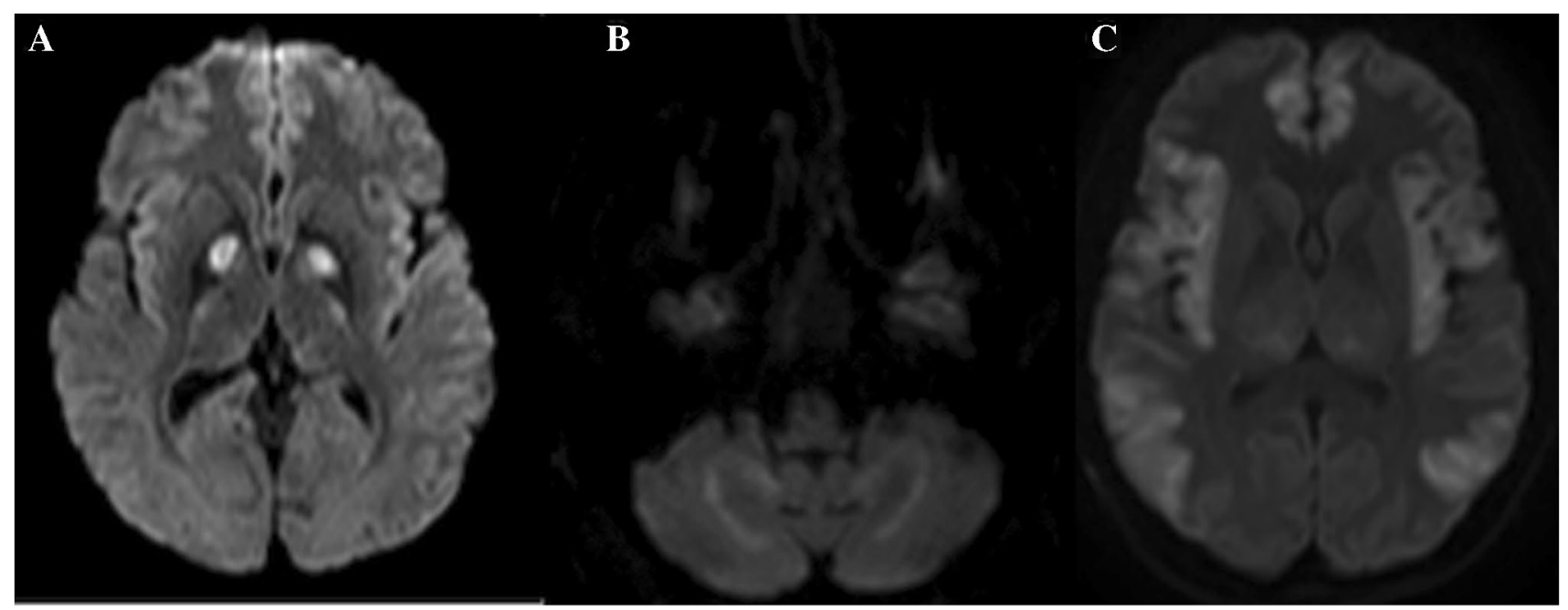

Fig. 6 A 29-year-old male with CO poisoning. Diffusion restriction in the globus pallidus (A). A 63-year-old male with metronidazole toxicity: diffusion changes (B) within the dentate nucleus. A 23-year-old

of the internal capsule, and cingulate gyrus is classical for acute hepatic encephalopathy [59, 62].

In uremic encephalopathy, vasogenic edema particularly in the globus pallidus is common, so DWI hyper signal intensity usually is secondary to the T2 shine effect; however, in a subset of the patients, cytotoxic edema can occur in the central portions of the vasogenic edema with true diffusion restriction. On rare occasions, true restricted diffusion is present (Fig. 6C). There is no association between the presence of diffusion restriction and creatinine levels [63].

PRES is a complex condition with many underlying etiologies, including hypertension, collagen-vascular disease, male with hyperuremic encephalopathy. Extensive cytotoxic edema symmetrically in bilateral cerebral cortex $(\mathrm{C})$

infection, and treatment by immunosuppressive agents or cytotoxic medications. The common physiopathology of these causes is the endothelial dysfunction and subsequent vasogenic edema in the cortex and subcortical white matter. The most common locations involved are the parietooccipital lobes and posterior frontal cortex. PRES is usually associated with the bright signal on ADC and normal DWI (Fig. 7a) but interstitial edema on FLAIR(Fig. 7b). However, a small subset of patients develops severe secondary vasoconstriction, leading to cerebral infarcts and diffusion restriction. Patients with hemorrhagic
Fig. 7 Posterior reversible encephalopathy syndrome (PRES): no increased diffusion (A) with increased FLAIR (B) suggestive of vasogenic edema

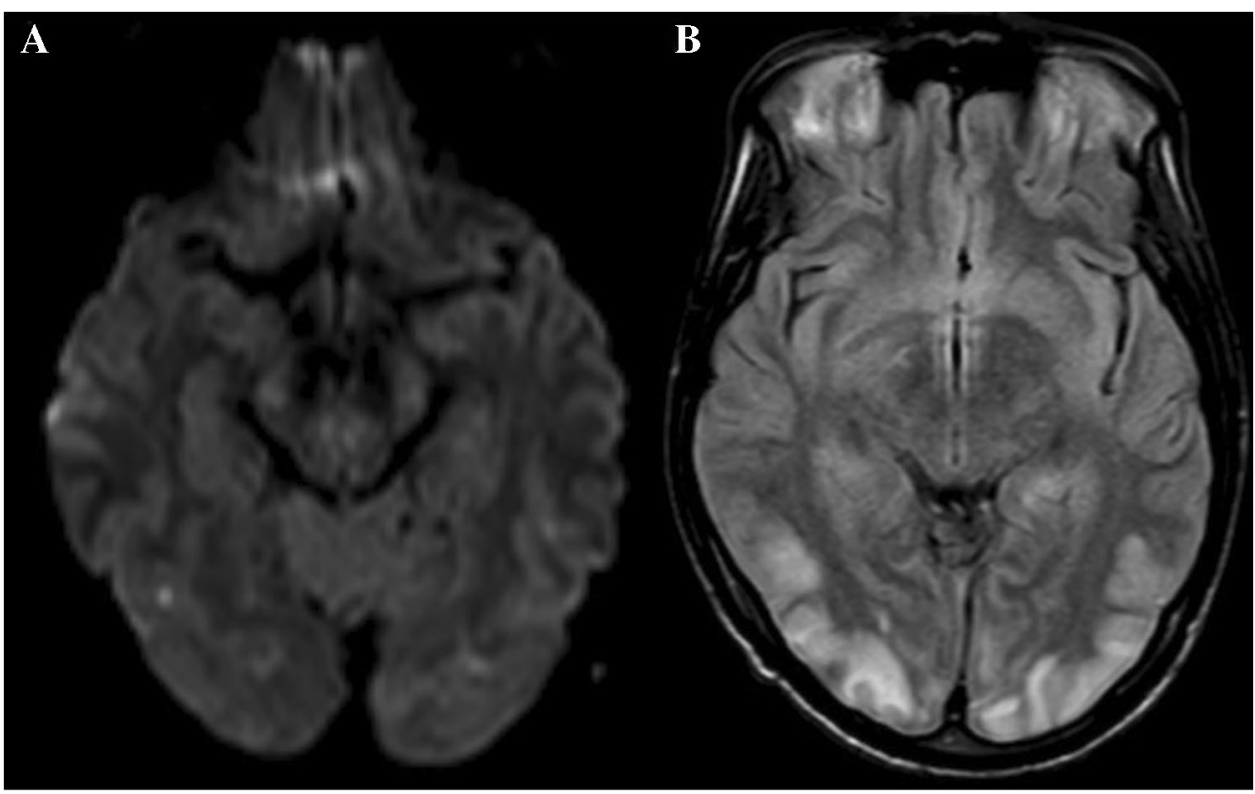


transformation and diffusion restriction have irreversible changes and tend to have poor clinical outcome [64].

In hypoglycemia, diffusion changes related to"excitotoxic" edema (increased extracellular amino acids provoking cytotoxic edema) can be seen in the internal capsules, hippocampi, and cerebral white matter in addition to the cerebral cortex (Fig. 8A and B). Diffusion changes can mimic ischemia and present in different patterns. Diffusion changes can present either with a low ADC (true restriction) or a normal ADC map (likely T2 shine through). In hypoglycemia, the DWI hyper signal intensities tend to be bilateral [65]. Early diffusion changes without FLAIR signal changes are usually associated with a favorable prognosis [66]. More extensive white matter changes and cortical involvement carry a less favorable prognosis. Hypoglycemic coma can mimic hypoxic injury but the appropriate history and white matter involvement clue in the diagnosis [67].

Osmotic demyelination (ODM) is caused by rapid hyponatremia correction and presents with decreased consciousness, seizures, and bulbar palsy, although presentation can be varied. There is a presumption that there is early cytotoxic edema within the myelin cells which is potentially reversible. As such, diffusion changes in the central pons
(Fig. 8C), basal ganglia, thalamus, cerebellum, and cerebral white matter may present within $24 \mathrm{~h}$ while signal changes on other sequences manifest later, and in some cases, after a few days.

Diffusion restriction usually resolves by 3 weeks after the symptom onset [68-70]. In comparison, the pontine involvement is quite specific for ODM; rare cases present with only extrapontine ODM. Clinical history in such cases is supportive of the diagnosis [71]. There is an association between the presence of the diffusion restriction in the basal ganglia and the severity of symptoms [68].

\section{Cytotoxic lesions of the corpus callosum}

The cytotoxic lesions of the corpus callosum (CLOCC) present secondary to many underlying neurological or systemic conditions such as infections, malignancy, medications, subarachnoid hemorrhage, or seizures. This condition has been known by a variety of terms including mild encephalopathy with reversible splenial lesions (MERS), reversible splenial lesion syndrome, reversible splenial lesions, transient splenial lesions, clinically silent lesions in the splenium of the
Fig. 8 White matter diffusion changes. A 50-year-old male with severe hypoglycemia. DWI was reversible. There is involvement of white matter $(\mathbf{A})$ and internal capsule (B) specific to hypoglycemia in addition to the cortical edema. A 61-year-old female with OMD (C). Reversible CLOCC: 25-year-old male with fever and high opening pressure on lumbar puncture. DWI (D) reveals restricted diffusion in the splenium of corpus callosum. Follow-up MRI 4 months later $(\mathbf{E})$ reveals normal corpus callosum

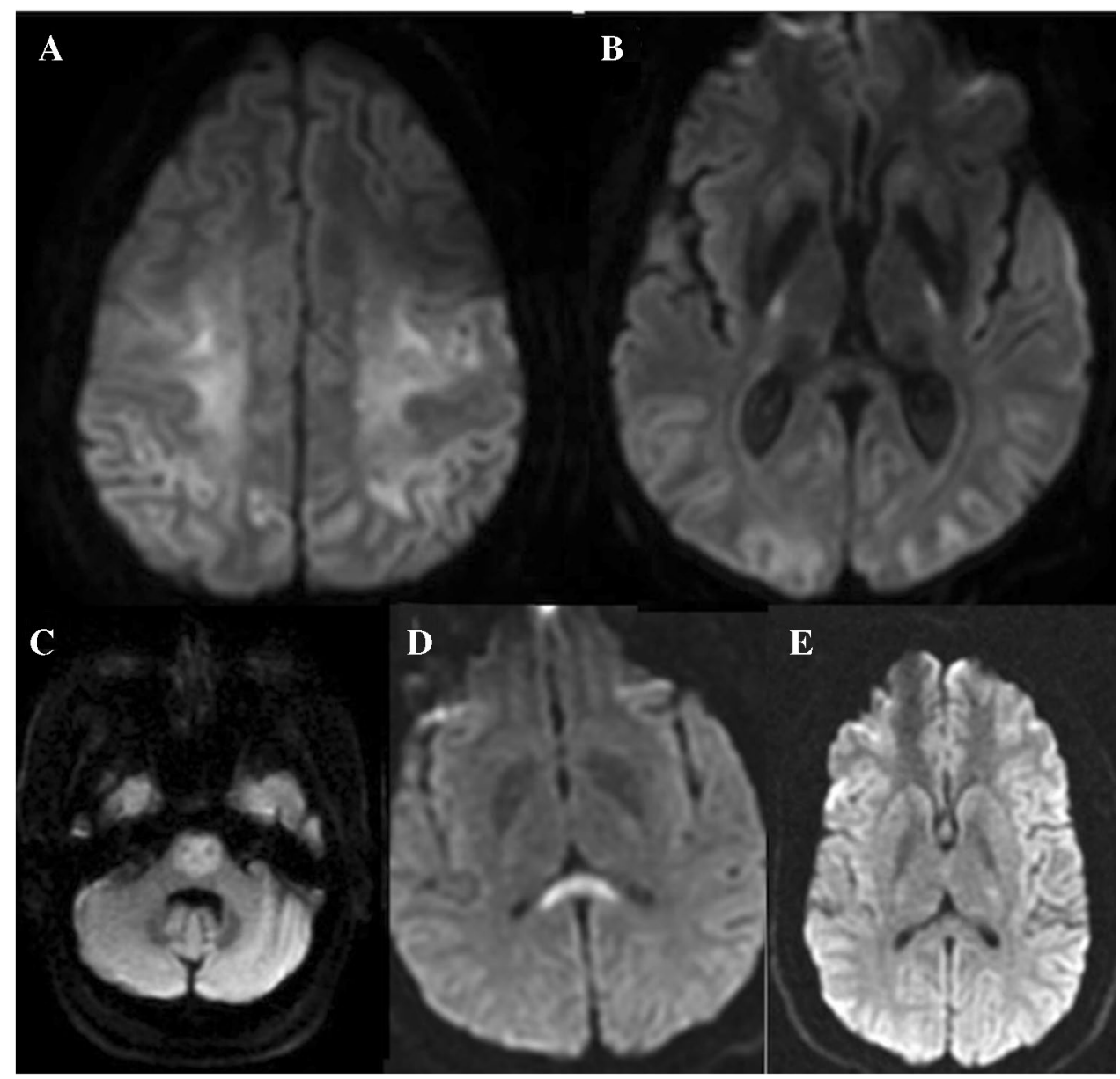


corpus callosum, and transient focal lesions in the splenium of the corpus callosum. These aforementioned stressors lead to increased extracellular cytokines and glutamates, which result in increased intracellular sodium and calcium levels promoting intracellular edema. The splenium is most vulnerable due to the increased concentration of glutamate. On imaging, there is diffusion restriction in the splenium of the corpus callosum (Fig. 8D). T2 and FLAIR signal changes are modest, but there is no mass effect or enhancement. Diffusion changes are reversible (Fig. 8E). It is pertinent to look for the underlying etiology if identifiable on imaging. Differential possibilities would include a posterior cerebral artery (PCA) territory infarct; therefore, any diffusion abnormalities in the PCA vascular distribution would favor ischemia [72].

\section{Post-seizure-related activity}

Diffusion restrictions can be observed in patients following single epileptic seizures but are more commonly seen after status epilepticus $[73,74]$. The findings are relevant to the location of ictal activity and are highly concordant with the electroclinical abnormalities [75]. A combination of DWI and EEG analysis can provide valuable information regarding seizure localization and propagation [76]. DWI hyper signal intensities are usually located ipsilateral to the epileptogenic lesions [76, 77]. Within the first $24 \mathrm{~h}$ after seizure onset, $4 \%$ of patients with an episode of seizure (excluding SE) exhibit diffusion abnormalities. The presence of the DWI signal alteration is associated with identifiable epileptogenic lesions on other sequences (compared to the group with no DWI findings) [73]. DWI/ $\mathrm{ADC}$ and arterial spin labeling perfusion imaging (ASL) are the most sensitive MR techniques in the depiction of changes secondary to epilepsy, but both techniques have lower sensitivity than electroencephalogram (EEG) [78].

DWI and FLAIR hyper signal intensity usually happens in both cortex and subcortical white matter (Fig. 9A and B). Isolated gray matter or white matter DWI hyper signal intensity is less common [77]. The prevalence of the DWI hyper signal intensity has been reported in up to $85 \%$ of patients with status epilepticus [76]. Posterior cerebral regions (parieto-occipital), hippocampi, and pulvinar regions are the most common locations with DWI hyper signal intensities. In SE, diffusion restriction of the hippocampus and the pulvinar is common, but these regions are not necessarily seizure onset locations [76]. These DWI and FLAIR signal changes are reversible (Fig. 9C).

Miscellaneous conditions DWI abnormalities are identified in many other conditions, and it is prudent that the reader be aware of other various presentations. Diffusion tensor imaging (DTI) can assess microstructural changes in many neurodegenerative conditions even before MRI changes appear. The review of these changes on DTI is beyond the scope of this manuscript. DWI has been shown to demonstrate increased diffusion in involved regions on DTI. DWI of the putamen can discriminate between idiopathic Parkinson's disease (PD) from atypical Parkinson's disease (APD), such as progressive supranuclear palsy and corticobasal degeneration, where it has been shown to have a higher ADC value in APD [79]. Similar increased ADC values are seen in the brain stem, optic radiations, and cerebellar peduncles in patients with Friedreich's ataxia [80].

Wallerian degeneration typically begins after 2 weeks in the distal white matter tracks after a known acute event such as stroke. Wallerian degeneration can present with diffusion restriction and absent or $\mathrm{T} 2$ hypointense signal changes in

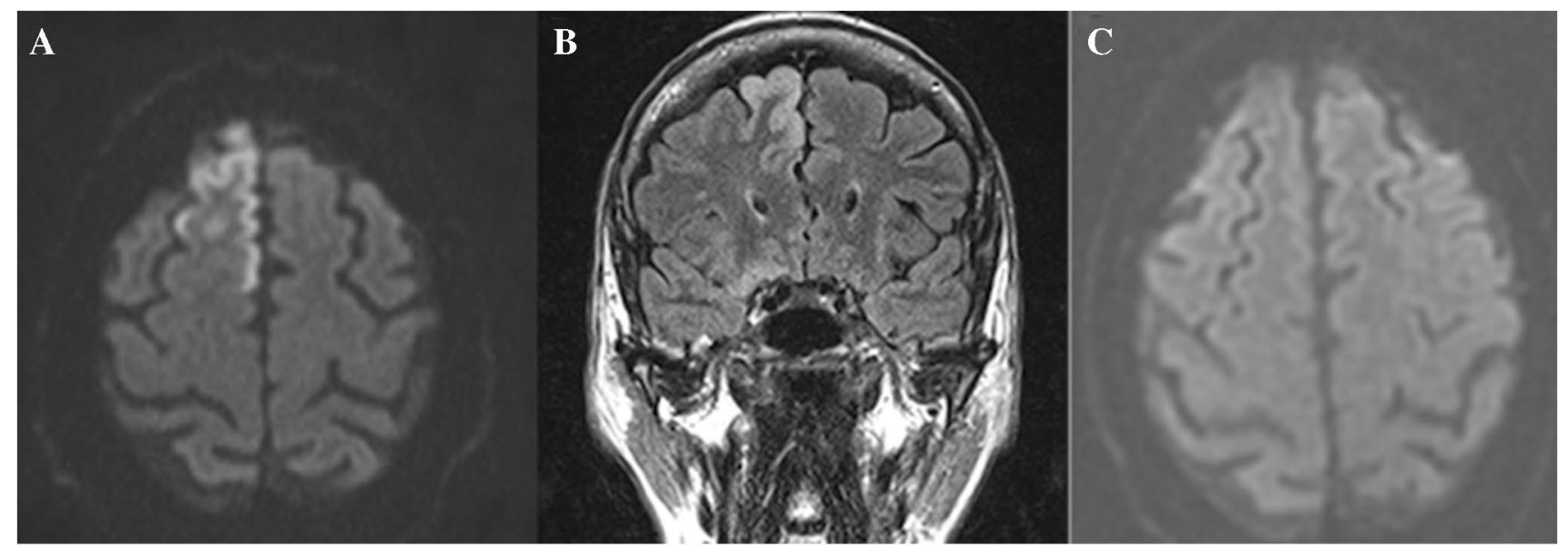

Fig. 9 Transient seizure related cytotoxic edema: Abnormal diffusion (A) and FLAIR (B) signal immediately noticed after recent seizures but resolved on follow up MRI (C) 
the early phase followed by $\mathrm{T} 2$ signal changes. These early changes are felt to be due to intramyelinic edema [81].

In patients with cerebral amyloid angiopathy (CAA), incidental DWI lesions in the cortex were associated with chronic cortical microinfarcts (CMI) and superficial siderosis on follow-up imaging, which are very unlikely to undergo cortical hemorrhagic transformation [82]. DWI lesions can provide a surrogate marker to the extent of CMI, which is associated with greater cognitive dysfunction. CAA can be associated with vasculitis, which is termed CAA-related inflammation (CAA-ri). DWI changes are more likely to be associated with CAA-ri [83].

DWI is of value in patients in idiopathic intracranial hypertension where bilateral optic nerve head DWI restriction has been correlated with papilledema. While this finding is specific, it only has modest sensitivity [84].

\section{Brain tumors}

Brain tumors can show varying degrees of diffusion changes. Diffusion changes reflect the cellularity of the tumor and the nucleus-cytoplasmic ratio. In general, the higher grade tumors have low ADC values.

Astrocytomas display heterogeneous diffusion signals, which is attributed to the varying cellularity and grade, and there is restricted diffusion in the regions of more aggressive tumors (Fig. 10A and B).

ADC values alone or in the combination of MR perfusion parameters relative cerebral blood volume (rCBV) can accurately grade gliomas [85]. ADC value of grade II astrocytoma (average of $1.299 \pm 0.294$ ) is significantly different than grade III (average of $0.929 \pm 0.17$ ). The average ADC value of grade III is significantly different than grade IV (average of $0.79 \pm 0.17$ ) [85]. In addition, the recent metaanalysis by Wang et al. has indicated the high accuracy of DWI/ADC to differentiate low-grade glioma (grade I and II) from high-grade glioma (III and IV) with an area under the curve (AUC) of 0.91 [86].

The magnetic field and the $b$ values have a significant impact on the accuracy with higher magnetic fields and higher $b$ values (3000 versus 1000) associated with higher accuracy [86]. Early evidence also suggests the ability of ADC values in the prediction of the genetic status in glioma including the glial fibrillary acidic protein, topoisomerase II $\alpha$ (Topo II $\alpha$ ) [85]. Diffusion imaging can predict the progression of both treated and untreated. Astrocytomas with progression of diffusion restriction are likely associated with recurrence and progression. In the same manner, increased diffusion on serial follow-up correlates with decreased tumor burden. ADC values also can differentiate tumor progression $(0.75-1.30$, mean $1.14 \pm 0.18)$ from post-radiation changes (1.29-2.06, mean $1.57 \pm 0.35)$ [87].

Diffusion changes can extend beyond the tumoral enhancing regions, reflecting the infiltrative spread pattern, typically of glioblastoma multiforme and astrocytoma (Fig. 11A and B). These perimarginal diffusion changes can differentiate glial tumors from solitary metastasis. One caveat to remember is that the resection cavity's margins in the immediate postoperative period can demonstrate restricted diffusion from infarction. In such circumstances, diffusion changes evolve or normalize [88].

Primary CNS lymphoma (PCNSL) presents as a solid or ring-enhancing lesion. PCNSL has the lowest ADC values (Fig. 12) compared to other brain tumors such as metastasis
Fig. 10 Infiltrating GBM demonstrating mild restricted diffusion with increased diffusion in the surrounding edema on DWI (A) and $\mathrm{ADC}(\mathbf{B})$

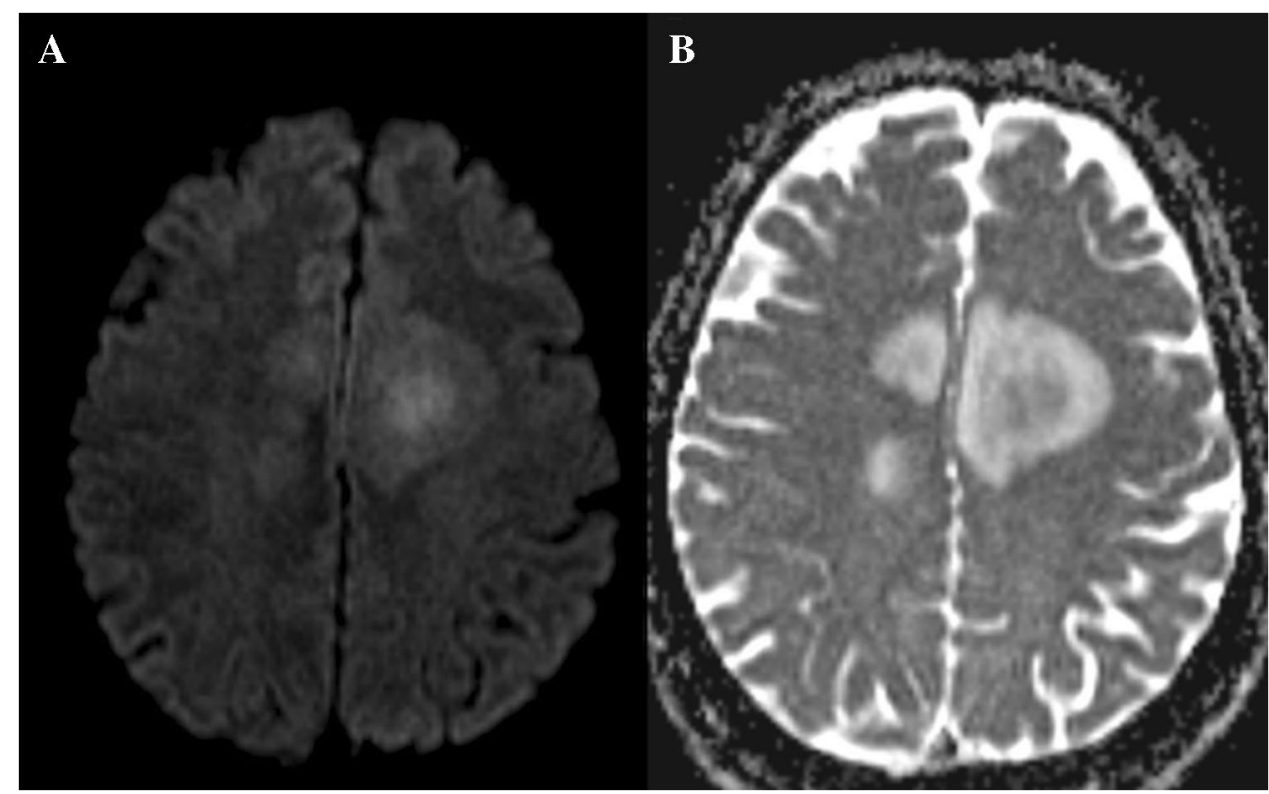


Fig. 11 A 23-year-old male with mostly necrotic GBM demonstrating increased diffusion. However, there is restricted diffusion medial to the lesion (arrows in $\mathbf{A}$ and $\mathbf{B}$ ) suggesting tumoral infiltration. Other necrotic tumors such as metastasis do not demonstrate restricted diffusion extending into surrounding white matter

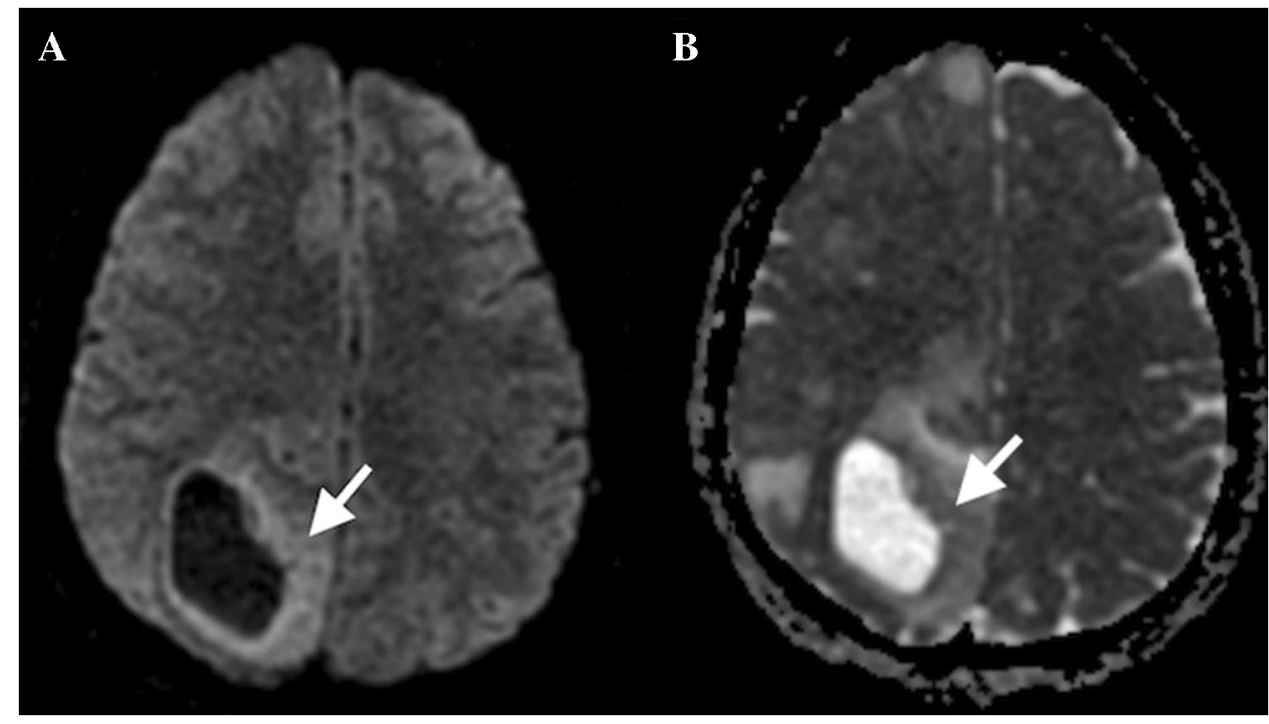

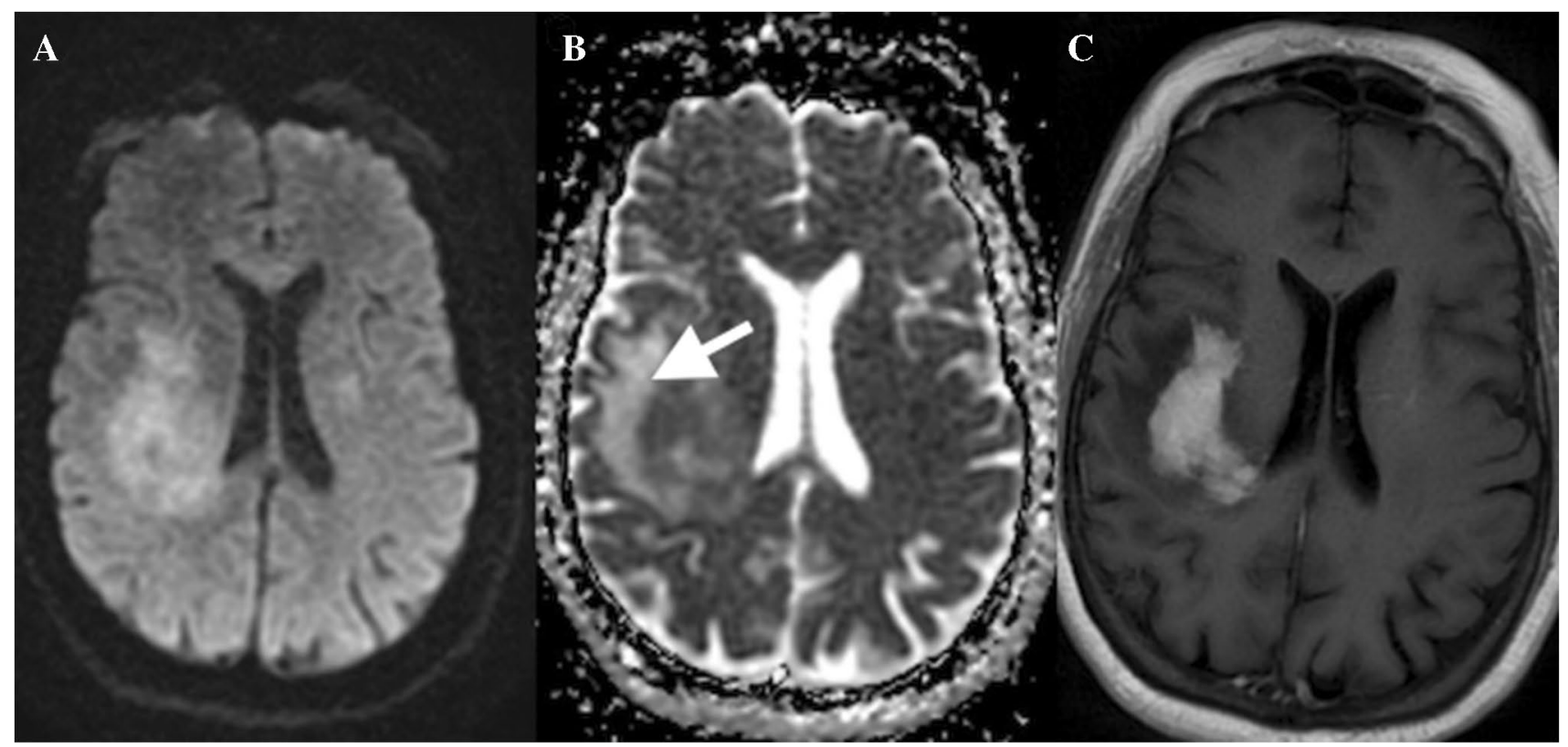

Fig. 12 A 65-year-old female with PCNSL: restricted diffusion (A and $\mathbf{B})$ corresponds to enhancing tumor (C), non-enhancing edema demonstrates increased diffusion on ADC (arrows in B)

or GBM. This is due to the high cellularity, decreased interstitial spaces, and greater nucleus to cytoplasmic ratio. Average ADC values in PCNSL are $0.73 \pm 0.13 \times 10^{-3} \mathrm{~mm}^{2} / \mathrm{s}$ compared to 1.02 and $1.03 \times 10^{-3} \mathrm{~mm}^{2} / \mathrm{sec}$ for $\mathrm{GBM}$ and metastasis, respectively [88]. Diffusion changes are homogeneous in PCNSL compared to other similar aggressive brain tumors. It has been shown that there are significant correlations between ADC value and the cellular Ki-67 expression in biopsied portions of PCNSL, the low ADC values to be indicative of increased proliferative activity of PCNSL [89].

DWI has shown to be helpful in differentiating pediatric posterior fossa tumors. Medulloblastoma, juvenile pilocytic astrocytoma (JPA), ependymoma, and brain stem glioma are common pediatric posterior fossa tumors. Medulloblastoma histologically contains more compact sheets of cells and high cellularity, which demonstrates low ADC compared to other tumors. Mean ADC values have been shown to be significantly different between the four groups of posterior fossa tumors in children. Mean ADC values were as follows: JPA $\left(1.63 \pm 0.05 \times 10^{-3}\right)$, medulloblastoma $\left(0.73 \pm 0.06 \times 10^{-3}\right)$, ependymoma $\left(1.15 \pm 0.07 \times 10^{-3}\right)$, and brain stem glioma $\left(1.37 \pm 0.12 \times 10^{-3}\right)$, with a $p$ value $<$ of 0.05 [90] 


\section{Future directions}

Diffusion tensor imaging is a well-established DWI technique utilizing the anisotropic nature of water diffusion to track white matter fibers. However, DTI is limited in mapping tracts crossing each other and with curved tracts. High angular resolution diffusion imaging (HARDI) was introduced to offset these challenges, which provide more detailed assessment of tracks [91]

While DWI assumes there is a Gaussian diffusion, based on likely distribution in a bell curve, diffusion kurtosis imaging (DKI) is based on the non-Gaussian distribution, providing more accurate measurement of diffusion. DKI has been shown to have clinical applications in stroke, TBI, brain neoplasm, neurodegenerative disorders, and demyelination [92]. The detailed techniques of these advancements are beyond the scope of this manuscript, but interested readers are encouraged to refer to the references for more details.

Radiomics incorporates artificial intelligence (AI) in brain tumors such as GBM assessment using computerized data from images that provide subvisual radiological cues. Radiomics information correlates with genomic information providing information on tumor response to therapy [93]. Initial research has shown that both diffusion and perfusion imaging data included in radiomics provide better prognostication of newly diagnosed GBM [94].

\section{Conclusion}

Many pathologies beyond stroke create diffusion restriction either by cytotoxic edema or by compromising the interstitial spaces within the brain. The resulting DWI characteristics can help differentiate various pathologies. DWI has proven to be dynamic in many pathologies, and the evolution of diffusion changes can prognosticate or characterize the underlying pathology.

Acknowledgements The authors would like to thank Dr. Cody B. Smith (Department of Radiology, Veterans Affairs Medical Center, University of Alabama at Birmingham, Birmingham, AL, USA) for his contribution for reviewing the manuscript for spell check and grammar check.

Author contributions SG: First Author and Corresponding Author Conceptualization, Writing original draft, Reviewing, Editing; OS: Co-First Author Writing original draft, Reviewing, Editing; HS: Conceptualization, Writing original draft, Reviewing, Editing; AS: Writing original draft, Reviewing, Editing; AR: Writing original draft, Reviewing, Editing; ZS: Writing original draft, Reviewing, Editing; AS: Conceptualization, Writing original draft, Reviewing, Editing; Revision

\section{Declarations}

Ethical approval All procedures performed in the studies involving human participants were in accordance with the ethical standards of the institutional and/or national research committee and with the 1964 Helsinki Declaration and its later amendments or comparable ethical standards.

Informed consent This is a review article, and no patient health information were included in this study. Sample educational figures are all anonymized.

Conflict of interest The authors declare that the research was conducted in the absence of any commercial or financial relationships that could be construed as a potential conflict of interest.

\section{References}

1. Castillo M (2014) History and evolution of brain tumor imaging: insights through radiology. Radiology 273:S111-S125

2. Le Bihan D, Breton E, Lallemand D et al (1986) MR imaging of intravoxel incoherent motions: application to diffusion and perfusion in neurologic disorders. Radiology 161:401-407. https://doi. org/10.1148/radiology.161.2.3763909

3. Balyasnikova S, Löfgren J, de Nijs R et al (2012) PET/MR in oncology: an introduction with focus on MR and future perspectives for hybrid imaging. Am J Nucl Med Mol Imaging 2:458-474

4. Hirata K, Nakaura T, Okuaki T et al (2018) Comparison of the image quality of turbo spin echo- and echo-planar diffusion-weighted images of the oral cavity. Medicine (Baltimore) 97(19):Med (United States) 97. https://doi.org/10.1097/MD.00000 00000010447

5. Yoshizako T, Yoshida R, Asou H et al (2021) Comparison between turbo spin-echo and echo planar diffusion-weighted imaging of the female pelvis with 3T MRI. Acta Radiol Open 10:205846012199473. https://doi.org/10.1177/2058460121 994737

6. Hagmann P, Jonasson L, Maeder P et al (2006) Understanding diffusion MR imaging techniques: from scalar diffusion-weighted imaging to diffusion tensor imaging and beyond. Radiographics 26 Suppl. https://doi.org/10.1148/rg.26si065510

7. Higaki T, Nakamura Y, Tatsugami $F$ et al (2018) Introduction to the technical aspects of computed diffusion-weighted imaging for radiologists. Radiographics 38:1131-1144. https://doi.org/10. 1148/rg.2018170115

8. Sener RN (2001) Diffusion MRI: apparent diffusion coefficient (ADC) values in the normal brain and a classification of brain disorders based on ADC values. Comput Med Imaging Graph 25:299-326. https://doi.org/10.1016/S0895-6111(00)00083-5

9. Schönfeld MH, Ritzel RM, Kemmling A et al (2018) Improved detectability of acute and subacute brainstem infarctions by combining standard axial and thin-sliced sagittal DWI. https://doi.org/ 10.1371/JOURNAL.PONE.0200092

10. Steffen P, Beyer L-S, McDonough R et al (2021) Improved detectability of brain stem ischemia by combining axial and coronal diffusion-weighted imaging. Stroke 52(5):1843-1846. https://doi. org/10.1161/STROKEAHA.120.032457

11. Oner AY, Celik H, Tali T et al (2007) Thin-section diffusionweighted magnetic resonance imaging of the brain with parallel imaging. Acta radiol 48:456-463. https://doi.org/10.1080/02841 850701297506 
12. Drake-Pérez M, Boto J, Fitsiori A et al (2018) Clinical applications of diffusion weighted imaging in neuroradiology. Insights Imaging 9:535-547

13. Chang SC, Lai PH, Chen WL et al (2002) Diffusion-weighted MRI features of brain abscess and cystic or necrotic brain tumors: comparison with conventional MRI. Clin Imaging 26:227-236. https://doi.org/10.1016/S0899-7071(02)00436-9

14. Toh CH, Wei KC, Ng SH et al (2011) Differentiation of brain abscesses from necrotic glioblastomas and cystic metastatic brain tumors with diffusion tensor imaging. Am J Neuroradiol 32:16461651. https://doi.org/10.3174/ajnr.A2581

15. Cartes-Zumelzu FW, Stavrou I, Castillo M et al (2004) Diffusionweighted imaging in the assessment of brain abscesses therapy. Am J Neuroradiol 25:1310-1317

16. Lotan E, Hoffmann C, Fardman A et al (2016) Postoperative versus spontaneous intracranial abscess: diagnostic value of the apparent diffusion coefficient for accurate assessment. Radiology 281:168-174. https://doi.org/10.1148/radiol.2016151708

17. Fukui MB, Williams RL, Mudigonda S (2001) CT and mr imaging features of pyogenic ventriculitis. AJNR Am J Neuroradiol 22(8):1510-1516

18. Rana S, Albayram S, Lin DDM, Yousem DM (2002) Diffusionweighted imaging and apparent diffusion coefficient maps in a case of intracerebral abscess with ventricular extension. AJNR Am J Neuroradiol 23(1):109-112

19. Fujikawa A, Tsuchiya K, Honya K, Nitatori T (2006) Comparison of MRI sequences to detect ventriculitis. Am J Roentgenol 187:1048-1053. https://doi.org/10.2214/AJR.04.1923

20. Mohan S, Jain KK, Arabi M, Shah GV (2012) Imaging of Meningitis and Ventriculitis. Neuroimaging Clin N Am 22:557-583

21. Mathews VP, Smith RR (1992) Choroid plexus infections: neuroimaging appearances of four cases. AJNR Am J Neuroradiol 13(1):374-378

22. Luthra G, Parihar A, Nath K et al (2007) Comparative evaluation of fungal, tubercular, and pyogenic brain abscesses with conventional and diffusion MR imaging and proton MR spectroscopy. Am J Neuroradiol 28:1332-1338. https://doi.org/10.3174/ajnr. A0548

23. Mueller-Mang C, Castillo M, Mang TG et al (2007) Fungal versus bacterial brain abscesses: is diffusion-weighted MR imaging a useful tool in the differential diagnosis? Neuroradiology 49:651-657

24. Schroeder PC, Post MJD, Oschatz E et al (2006) Analysis of the utility of diffusion-weighted MRI and apparent diffusion coefficient values in distinguishing central nervous system toxoplasmosis from lymphoma. Neuroradiology 48:715-720. https://doi. org/10.1007/s00234-006-0123-y

25. Kumar GGS, Mahadevan A, Guruprasad AS et al (2010) Eccentric target sign in cerebral toxoplasmosis: neuropathological correlate to the imaging feature. J Magn Reson Imaging 31:1469-1472. https://doi.org/10.1002/JMRI.22192

26. Kimura-Hayama ET, Higuera JA, Corona-Cedillo R et al (2010) Neurocysticercosis: radiologic-pathologic correlation. Radiographics 30:1705-1719. https://doi.org/10.1148/rg.306105522

27. Santos GT, Leite CC, Machado LR et al (2013) Reduced diffusion in neurocysticercosis: circumstances of appearance and possible natural history implications. Am J Neuroradiol 34:310-316. https://doi.org/10.3174/ajnr.A3198

28. Fragoso DC, da Mota Gonçalves Filho AL, Pacheco FT et al (2017) Imaging of Creutzfeldt-Jakob disease: imaging patterns and their differential diagnosis. Radiographics 37:234-257. https://doi.org/10.1148/rg.2017160075

29. Manix M, Kalakoti P, Henry M et al (2015) Creutzfeldt-Jakob disease: updated diagnostic criteria, treatment algorithm, and the utility of brain biopsy. Neurosurg Focus 39(5):E2. https://doi.org/ 10.3171/2015.8.FOCUS15328

30. Vitali P, MacCagnano E, Caverzasi E et al (2011) Diffusionweighted MRI hyperintensity patterns differentiate CJD from other rapid dementias. Neurology 76:1711-1719. https://doi.org/ 10.1212/WNL.0b013e31821a4439

31. Rudge P, Hyare H, Green A et al (2018) Imaging and CSF analyses effectively distinguish CJD from its mimics. J Neurol Neurosurg Psychiatry 89:461-466. https://doi.org/10.1136/ jnnp-2017-316853

32. Ellul M, Solomon T (2018) Acute encephalitis - diagnosis and management. Clin Med J R Coll Physicians London 18:155-159

33. Sotoudeh H, Razaei A, Saadatpour Z et al (2020) Brainstem encephalitis. The role of imaging in diagnosis. Curr Probl Diagn Radiol S0363-0188(20):30177-30178

34. Fillatre P, Crabol Y, Morand P et al (2017) Encéphalite infectieuse : diagnostic étiologique non fait à 48 heures, conduite à tenir. Med Mal Infect 47:236-251

35. Bridwell R, Long B, Gottlieb M (2020) Neurologic complications of COVID-19. Am J Emerg Med 38:1549.e3-1549.e7. https://doi. org/10.1016/j.ajem.2020.05.024

36. Lian ZY, Huang B, He SR et al (2012) Diffusion-weighted imaging in the diagnosis of enterovirus 71 encephalitis. Acta Radiol 53:208-213. https://doi.org/10.1258/ar.2011.110407

37. Prakash M, Kumar S, Gupta RK (2004) Diffusion-weighted MR imaging in Japanese encephalitis. J Comput Assist Tomogr 28:756-761. https://doi.org/10.1097/00004728-200411000-00005

38. Tsuchiya K, Katase S, Yoshino A, Hachiya J (1999) Diffusion-weighted MR imaging of encephalitis. Am J Roentgenol 173:1097-1099. https://doi.org/10.2214/ajr.173.4.10511186

39. Kiroğlu Y, Calli C, Yunten N et al (2006) Diffusion-weighted MR imaging of viral encephalitis. Neuroradiology 48:875-880. https:// doi.org/10.1007/s00234-006-0143-7

40. Küker W, Nägele T, Schmidt F et al (2004) Diffusionweighted MRI in herpes simplex encephalitis: a report of three cases. Neuroradiology 46:122-125. https://doi.org/10.1007/ s00234-003-1145-3

41. Chatzikonstantinou A, Szabo K, Ottomeyer C et al (2009) Successive affection of bilateral temporomesial structures in a case of non-paraneoplastic limbic encephalitis demonstrated by serial MRI and FDG-PET. J Neurol 256:1753-1755

42. Thuerl C, Müller K, Laubenberger J et al (2003) MR imaging of autopsy-proved paraneoplastic limbic encephalitis in non-hodgkin lymphoma. AJNR Am J Neuroradiol 24(3):507-511

43. Urbach H, Soeder BM, Jeub M et al (2006) Serial MRI of limbic encephalitis. Neuroradiology 48:380-386. https://doi.org/10.1007/ s00234-006-0069-0

44. Hou DJ, Tong KA, Ashwal S et al (2007) Diffusion-weighted magnetic resonance imaging improves outcome prediction in adult traumatic brain injury. J Neurotrauma 24:1558-1569. https://doi. org/10.1089/neu.2007.0339

45. Shakir A, Aksoy D, Mlynash M et al (2016) Prognostic value of quantitative diffusion-weighted mri in patients with traumatic brain injury. J Neuroimaging 26:103-108. https://doi.org/10.1111/ jon. 12286

46. Elliott CA, Ramaswamy V, Jacob FD et al (2017) Early diffusion restriction of white matter in infants with small subdural hematomas is associated with delayed atrophy. Child's Nerv Syst 33:289-295. https://doi.org/10.1007/s00381-016-3271-3

47. Huisman TAGM, Sorensen AG, Hergan K et al (2003) Diffusionweighted imaging for the evaluation of diffuse axonal injury in closed head injury. J Comput Assist Tomogr 27:5-11. https://doi. org/10.1097/00004728-200301000-00002

48. Moen KG, Brezova V, Skandsen T et al (2014) Traumatic axonal injury: the prognostic value of lesion load in corpus callosum, 
brain stem, and thalamus in different magnetic resonance imaging sequences. J Neurotrauma 31:1486-1496. https://doi.org/10.1089/ neu.2013.3258

49. Gasparetto EL, Rueda Lopes FC, Domingues RC, Domingues RC (2011) Diffusion imaging in traumatic brain injury. Neuroimaging Clin N Am 21:115-125

50. Kuo K-H, Pan Y-J, Lai Y-J et al (2014) Dynamic MR imaging patterns of cerebral fat embolism: a systematic review with illustrative cases. Am J Neuroradiol 35:1052-1057. https://doi.org/10. 3174/AJNR.A3605

51. Abou Zeid N, Pirko I, Erickson B et al (2012) Diffusion-weighted imaging characteristics of biopsy-proven demyelinating brain lesions. Neurology 78:1655-1662. https://doi.org/10.1212/WNL. 0b013e3182574f66

52. Koelblinger C, Fruehwald-Pallamar J, Kubin K et al (2013) Atypical idiopathic inflammatory demyelinating lesions (IIDL): conventional and diffusion-weighted MR imaging (DWI) findings in 42 cases. Eur J Radiol 82:1996-2004. https://doi.org/10.1016/j. ejrad.2013.07.026

53. Rueda-Lopes FC, Hygino Da Cruz LC, Doring TM, Gasparetto EL (2014) Diffusion-weighted imaging and demyelinating diseases: new aspects of an old advanced sequence. AJR Am J Roentgenol 202(1):W34-42. https://doi.org/10.2214/AJR.13.11400

54. Mabray MC, Cohen BA, Villanueva-Meyer JE et al (2015) Performance of apparent diffusion coefficient values and conventional MRI features in differentiating tumefactive demyelinating lesions from primary brain neoplasms. Am J Roentgenol 205:1075-1085. https://doi.org/10.2214/AJR.14.13970

55. Eisele P, Szabo K, Griebe M et al (2012) Reduced diffusion in a subset of acute MS lesions: a serial multiparametric MRI study. Am J Neuroradiol 33:1369-1373. https://doi.org/10.3174/ajnr. A2975

56. Davoudi Y, Foroughipour M, Torabi R et al (2016) Diffusion weighted imaging in acute attacks of multiple sclerosis. Iran J Radiol 13:1-5. https://doi.org/10.5812/iranjradiol.21740

57. Sung EK, Farris C, Abdalkader M, Mian A (2018) Acute neurologic syndromes beyond stroke: the role of emergent mr imaging. Neuroimaging Clin N Am 28:375-395

58. Rovira Cañellas A, Alonso J, Córdoba J (2008) MR imaging findings in hepatic encephalopathy. Am J Neuroradiol 29:1612-1621

59. McKinney AM, Lohman BD, Sarikaya B et al (2010) Acute hepatic encephalopathy: diffusion-weighted and fluid-attenuated inversion recovery findings, and correlation with plasma ammonia level and clinical outcome. Am J Neuroradiol 31:1471-1479. https://doi.org/10.3174/ajnr.A2112

60. Rosario M, Mcmahon K, Finelli PF (2013) Diffusion-weighted imaging in acute hyperammonemic encephalopathy. Neurohospitalist 3:125-130. https://doi.org/10.1177/1941874412467806

61. U-King-Im JM, Yu E, Bartlett E et al (2011) Acute hyperammonemic encephalopathy in adults: imaging findings. Am J Neuroradiol 32:413-418. https://doi.org/10.3174/ajnr.A2290

62. Hegde AN, Mohan S, Lath N, Lim CCT (2011) Differential diagnosis for bilateral abnormalities of the basal ganglia and thalamus. Radiographics 31(1):5. https://doi.org/10.1148/rg.311105041

63. Kim DM, Lee IH, Song CJ (2016) Uremic encephalopathy: MR imaging findings and clinical correlation. Am J Neuroradiol 37:1604-1609. https://doi.org/10.3174/ajnr.A4776

64. Tetsuka S, Ogawa T (2019) Posterior reversible encephalopathy syndrome: a review with emphasis on neuroimaging characteristics. J Neurol Sci 404:72-79

65. Kang EG, Jeon SJ, Choi SS et al (2010) Diffusion MR imaging of hypoglycemic encephalopathy. Am J Neuroradiol 31:559-564. https://doi.org/10.3174/ajnr.A1856

66. Adam G, Ferrier M, Patsoura S et al (2018) Magnetic resonance imaging of arterial stroke mimics: a pictorial review. Insights Imaging 9:815-831
67. Johkura K, Nakae Y, Kudo Y et al (2012) Early diffusion MR imaging findings and short-term outcome in comatose patients with hypoglycemia. Am J Neuroradiol 33:904-909. https://doi. org/10.3174/ajnr.A2903

68. Garg P, Aggarwal A, Malhotra R, Dhall S (2019) Osmotic demyelination syndrome - evolution of extrapontine before pontine myelinolysis on magnetic resonance imaging. J Neurosci Rural Pract 10:126-135. https://doi.org/10.4103/jnrp.jnrp_240_ 18

69. Ruzek KA, Campeau NG, Miller GM (2004) Early diagnosis of central pontine myelinolysis with diffusion-weighted imaging. Am J Neuroradiol 25:210-213

70. Cramer SC, Stegbauer KC, Schneider A et al (2001) Decreased diffusion in central pontine myelinolysis. AJNR Am J Neuroradiol 22(8):1476-1479

71. O'connor KM, Barest G, Moritani T et al (2013) Dazed and diffused: making sense of diffusion abnormalities in neurologic pathologies. Br J Radiol 86(1032):20130599. https://doi.org/10. 1259/bjr.20130599

72. Starkey J, Kobayashi N, Numaguchi Y, Moritani T (2017) Cytotoxic lesions of the corpus callosum that show restricted diffusion: mechanisms, causes, and manifestations. Radiographics 37:562-576. https://doi.org/10.1148/rg.2017160085

73. Hübers A, Thoma K, Schocke M et al (2018) Acute DWI reductions in patients after single epileptic seizures - more common than assumed. Front Neurol 9:550. https://doi.org/10.3389/fneur. 2018.00550

74. Wiest R, Beisteiner R (2019) Recent developments in imaging of epilepsy. Curr Opin Neurol 32:530-538

75. Di Bonaventura C, Bonini F, Fattouch J et al (2009) Diffusionweighted magnetic resonance imaging in patients with partial status epilepticus. Epilepsia 50:45-52. https://doi.org/10.1111/j. 1528-1167.2008.01970.x

76. Chatzikonstantinou A, Gass A, Förster A et al (2011) Features of acute DWI abnormalities related to status epilepticus. Epilepsy Res 97:45-51. https://doi.org/10.1016/j.eplepsyres.2011.07.002

77. Mendes A, Sampaio L (2016) Brain magnetic resonance in status epilepticus: a focused review. Seizure 38:63-67

78. Matsuura K, Maeda M, Okamoto K et al (2015) Usefulness of arterial spin-labeling images in periictal state diagnosis of epilepsy. J Neurol Sci 359:424-429. https://doi.org/10.1016/J.JNS. 2015.10.009

79. Rizzo G, Martinelli P, Manners D et al (2008) Diffusion-weighted brain imaging study of patients with clinical diagnosis of corticobasal degeneration, progressive supranuclear palsy and Parkinson's disease. Brain 131:2690-2700. https://doi.org/10.1093/ BRAIN/AWN195

80. Rizzo G, Tonon C, Valentino ML et al (2011) Brain diffusionweighted imaging in Friedreich's ataxia. Mov Disord 26:705-712. https://doi.org/10.1002/MDS.23518

81. Musson R, Romanowski C (2010) Restricted diffusion in Wallerian degeneration of the middle cerebellar peduncles following pontine infarction. Polish J Radiol 75:38

82. van Veluw SJ, Lauer A, Charidimou A et al (2017) Evolution of DWI lesions in cerebral amyloid angiopathy: evidence for ischemia. Neurology 89:2136. https://doi.org/10.1212/WNL. 0000000000004668

83. Renard D, Tatu L, Collombier L et al (2018) Cerebral amyloid angiopathy and cerebral amyloid angiopathy-related inflammation: comparison of hemorrhagic and DWI MRI features. J Alzheimer's Dis 64:1113-1121. https://doi.org/10.3233/JAD-180269

84. Viets R, Parsons M, Van SG et al (2013) Fellows' Journal Club: Hyperintense optic nerve heads on diffusion-weighted imaging: a potential imaging sign of papilledema. AJNR Am J Neuroradiol 34:1438. https://doi.org/10.3174/AJNR.A3388 
85. Qin JB, Zhang H, Wang XC et al (2019) Combination value of diffusion-weighted imaging and dynamic susceptibility contrastenhanced MRI in astrocytoma grading and correlation with GFAP, topoisomerase II $\alpha$ and MGMT. Oncol Lett 18:2763-2770. https:// doi.org/10.3892/ol.2019.10656

86. Wang Q, Lei D, Yuan Y, Xiong N (2020) Accuracy of ADC derived from DWI for differentiating high-grade from low-grade gliomas: systematic review and meta-analysis. Medicine (Baltimore) 99(8):e19254

87. Matsushita T, Anami D, Arioka T et al (2008) Basic study of susceptibility-weighted imaging at 1.5T. Acta Med Okayama 62:159-168

88. Neska-Matuszewska M, Bladowska J, Sąsiadek M, Zimny A (2018) Differentiation of glioblastoma multiforme, metastases and primary central nervous system lymphomas using multiparametric perfusion and diffusion MR imaging of a tumor core and a peritumoral zone-Searching for a practical approach. PLoS One 13:. https://doi.org/10.1371/journal.pone.0191341

89. Schob S, Meyer J, Gawlitza M et al (2016) Diffusion-weighted MRI reflects proliferative activity in primary CNS lymphoma. PLoS One 11(8):e0161386. https://doi.org/10.1371/journal.pone. 0161386

90. Mustafa WF, Abbas M (2020) Elsorougy L (2020) Role of diffusion-weighted imaging in differentiation between posterior fossa brain tumors. Egypt J Neurol Psychiatry Neurosurg 561(56):1-8. https://doi.org/10.1186/S41983-019-0145-0

91. Descoteaux M (2015) High angular resolution diffusion imaging (HARDI). J. Webster (ed.), Wiley Encyclopedia of Electrical and Electronics Engineering. Copyright \# 2015 John Wiley \& Sons, Inc. https://doi.org/10.1002/047134608X.W8258

92. Steven AJ, Zhuo J, Melhem ER (2014) Diffusion kurtosis imaging: an emerging technique for evaluating the microstructural environment of the brain. Am J Roentgenol 202:. https://doi.org/ 10.2214/AJR.13.11365

93. Singh G, Manjila S, Sakla N et al (2021) (2021) Radiomics and radiogenomics in gliomas: a contemporary update. Br J Cancer 1255(125):641-657. https://doi.org/10.1038/s41416-021-01387-w

94. Park JE, Kim HS, Jo Y et al (2020) Radiomics prognostication model in glioblastoma using diffusion- and perfusionweighted MRI. Sci Rep 10(1):4250. https://doi.org/10.1038/ s41598-020-61178-w

Publisher's note Springer Nature remains neutral with regard to jurisdictional claims in published maps and institutional affiliations. 